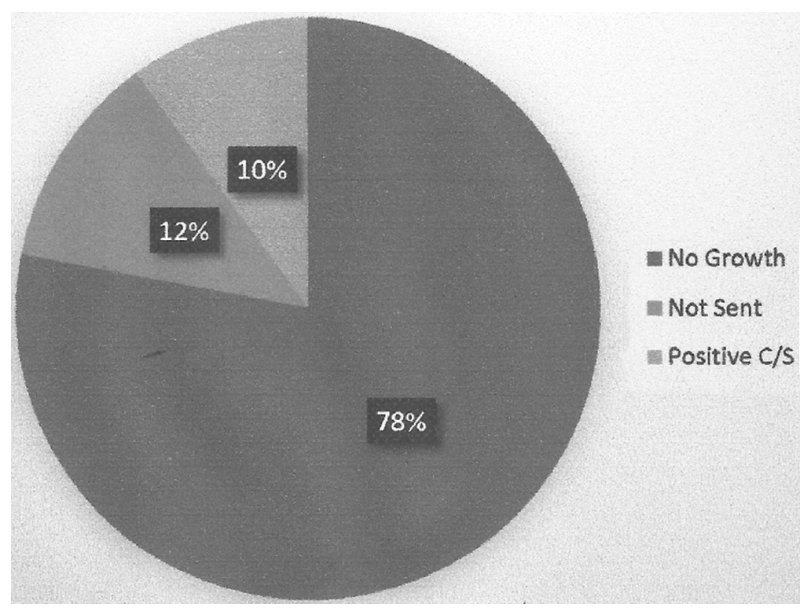

Abstract GP245 Figure 4 PICC linetip cultures

Results The charts of 41 Neonates with PICC lines, were reviewed. One hundred percent had a PICC Sticker inserted in the chart. Twenty-six 'PICC Stickers' (63\%) had 100\% compliance with all the 15 documentation criteria. Thirty-eight charts (92\%) had 11 or more documentation criteria completed. There was $100 \%$ compliance with Date, time, Indication, Catheter type, Insertion Depth, time of $\times$ ray, Position on $\times$ ray, line taped at, 'Line Suitable' and Clinical Signature. The Documentation sticker with less than 100\% compliance included catheter size 80\% (33/41), measured length 95\% (37/ 41 ), no change $75 \%(31 / 41)$ and with draw 95\%(39/41).

Formal radiological reports documented the PICC line tip position in forty of the forty-one $\times$ rays.

Kappa score for correlation between Paediatrician and Radiologist was $0.637(95 \%$ CI $0.394-0.880)$.

This audit demonstrates significant improvement to the standard of clinical documentation (as Shown in Bar Charts). Findings

\begin{tabular}{|c|c|c|c|c|c|}
\hline $\begin{array}{l}\text { Babies } \\
\text { 2015-2018 }\end{array}$ & $\begin{array}{l}\text { PICC Lines } \\
\text { 2015-2018 }\end{array}$ & $\begin{array}{l}\text { Mean } \\
\text { Gestation }\end{array}$ & $\begin{array}{l}\text { Mean } \\
\text { Weight }\end{array}$ & $\begin{array}{l}\text { Mean } \\
\text { Age at } \\
\text { Insertion }\end{array}$ & $\begin{array}{l}\text { Duration of } \\
\text { PICC Line }\end{array}$ \\
\hline 287 & 41 & $\begin{array}{l}31 \text { Weeks } \\
(26-35)\end{array}$ & $\begin{array}{l}1.2 \mathrm{Kg} \\
(0.6-1.98)\end{array}$ & $\begin{array}{l}28 \text { Hours } \\
(1-192)\end{array}$ & $\begin{array}{l}11.5 \text { Days } \\
(2-21)\end{array}$ \\
\hline
\end{tabular}

Conclusion This audit shows that, following the introduction of a 'CL Stickers' quality Improvement initiative, there has been significant improvement in quality of documentation of PICC lines in the past 4-year period. While kappa scores for inter-observer variation for the radiological position of the $\mathrm{CL}$ has shown some improved since 2014, it remains low at 0.637 .

\section{GP246 SPECIALISTS' ATTITUDES TO VACCINATION TODAY: RESULTS OF THE QUESTIONNAIRE SURVEY}

\footnotetext{
${ }^{1}$ Alla Petrova, ${ }^{1}$ Anastasiya Vanyarkina*, ${ }^{2}$ Svetlana Shugaeva, ${ }^{1}$ Lyubov Rychkova, ${ }^{1}$ Ekaterina Moskaleva. ${ }^{1}$ Scientific Centre for Family Health and Human Reproduction Problems, Irkutsk, Russian Federation; ${ }^{2}$ Irkutsk State Medical University, Irkutsk, Russian Federation
}

10.1136/archdischild-2019-epa.305
People's attitude towards immunization reflects not only understanding of efficacy and safety of preventive vaccination, but also confidence to the health care system in general. Current decreasing of the confidence level to vaccination in the European region (in 2017 this index reached 83\%) demands that specialists know how to improve and retain confidence to programs of immunization, and how to maintain accumulated experience of preventive vaccination.

Aim To assess the specialists' attitude and knowledge about vaccination.

Materials and Methods Sixty-two specialists, working at Irkutsk Perinatal Centre (III Level), participated in an anonymous questionnaire survey, conducted from November to December 2018. The original questionnaire contained 12 questions on demographic characteristics, attitude to vaccination (including personal confidence in vaccines), and sources specialists usually use to know about vaccination. The following specialists participated in the survey: neonatologists $(n=35)$, intensivists $(n=15)$, pediatricians $(n=7)$, obstetricians $(n=5)$. They belonged to following age groups: 21 to 30 years - 23 (37\%), 31 to 40 years $-24(38.7 \%), 41$ to 50 years -11 $(17.7 \%)$, and older 51 years $-4(6.6 \%)$.

Results All respondents considered vaccination as a safe and reliable method against infections. The majority $(n=44 ; 71 \%)$ believed it was necessary to recommend parents not only compulsory vaccination, included in the National Vaccination Schedule, but also immunization according epidemiological indications and vaccination against currently widespread infections. Many respondents $(\mathrm{n}=55 ; 88.7 \%)$ considered necessary to lawfully influence parents' decision not to vaccinate their children. More than half of specialists $(n=37 ; 60 \%)$ vaccinated their children with both mandatory and recommended vaccines. Sixty six point one percent of respondents considered their knowledge about vaccination insufficient. At the same time, knowledge about vaccination was lower in age groups from 21 to 30 years and from 31 to 40 years $(n=21$; $91.3 \%$ and $n=12 ; 50 \%$, respectively). However the analysis of dependence between specialties and knowledge in all age groups did not reveal significant differences $(p=0.6)$. Respondents received information about vaccination predominantly from reading specialized medical literature $(n=50 ; 82.6 \%)$, during participation in conferences and workshops $(n=39$; $63 \%)$, and using specialized web resources $(n=32 ; 51.6 \%)$.

Conclusion Despite having confidence in vaccination, many specialists notice the need to study this issue more thoroughly. Monitoring of doctors' awareness on vaccination, creation of up-to-date specialized education media resources on immunoprophylaxis will not only enhance knowledge of specialists, but also maintain the confidence in vaccination in patients, and reduce the number of ungrounded refusals.

\section{GP247 EFFICACY OF INTRAVENOUS IMMUNOGLOBULINS IN THE PROPHYLAXIS OF INFECTION IN PRETERM AND LOW BIRTH WEIGHT NEONATES: A SCIENTIFIC LITERATURE REVIEW}

${ }^{1}$ Cayla Bloomer*, ${ }^{2}$ Saad Sharif, ${ }^{3}$ Rizwan Khan, ${ }^{3}$ Niazzy Al Assaf. ${ }^{1}$ University of Limerick, Limerick, Ireland; ' ${ }^{2}$ University College Dublin, Dublin, Ireland; ${ }^{3}$ University Maternity Hospital Limerick, Limerick, Ireland

10.1136/archdischild-2019-epa.306

Background Despite recent advances in critical care, infection remains one of the leading causes of mortality and morbidity 